\title{
PARTICLE KNOTS IN TORIC MODULAR SPACE
}

\author{
J.A.de Wet
}

\section{Box 514,Plettenberg Bay,6600,South Africa}

Keywords: Compactification, Coxeter Graphs, GF(4),Torus Knots, QCD, Triality.

\begin{abstract}
The goal of this contribution is to relate quarks to knots or loops in a 6-space $\mathrm{CP}^{3}$ that then ollapses into a torus in real 3-space $\mathrm{P}^{3}$ instantaneously after the Big Bang, and massive inflation, when 3 quarks unite to form nucleons.

\section{Introduction}

Kedia et. al. in recent paper [10] investigate knotted structures in hydrod smmic ids su h as current-guiding magnetic field lines in a plasma, or vortex lines of classice or qu ntum 1 , which arise naturally as excitations that carry helicity that is a measure of the ted ness of the field. In particular their Fig.2g is a trefoil which is our Fig.3 without the gramup that $\mathrm{w} / \mathrm{l}$ be seen in Section 2 to collapse into a point) and the color-coding.
\end{abstract}

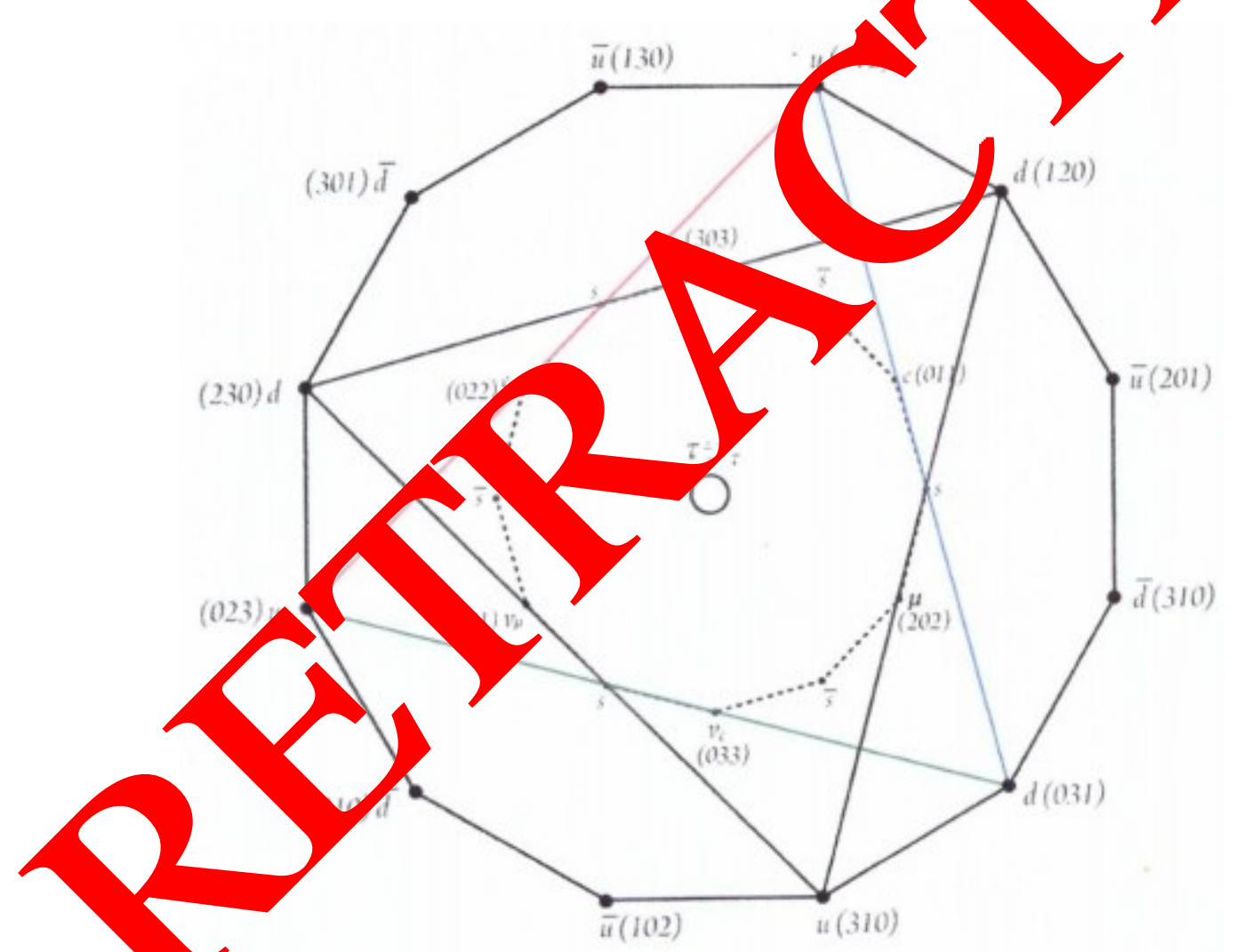

Fig. 1 


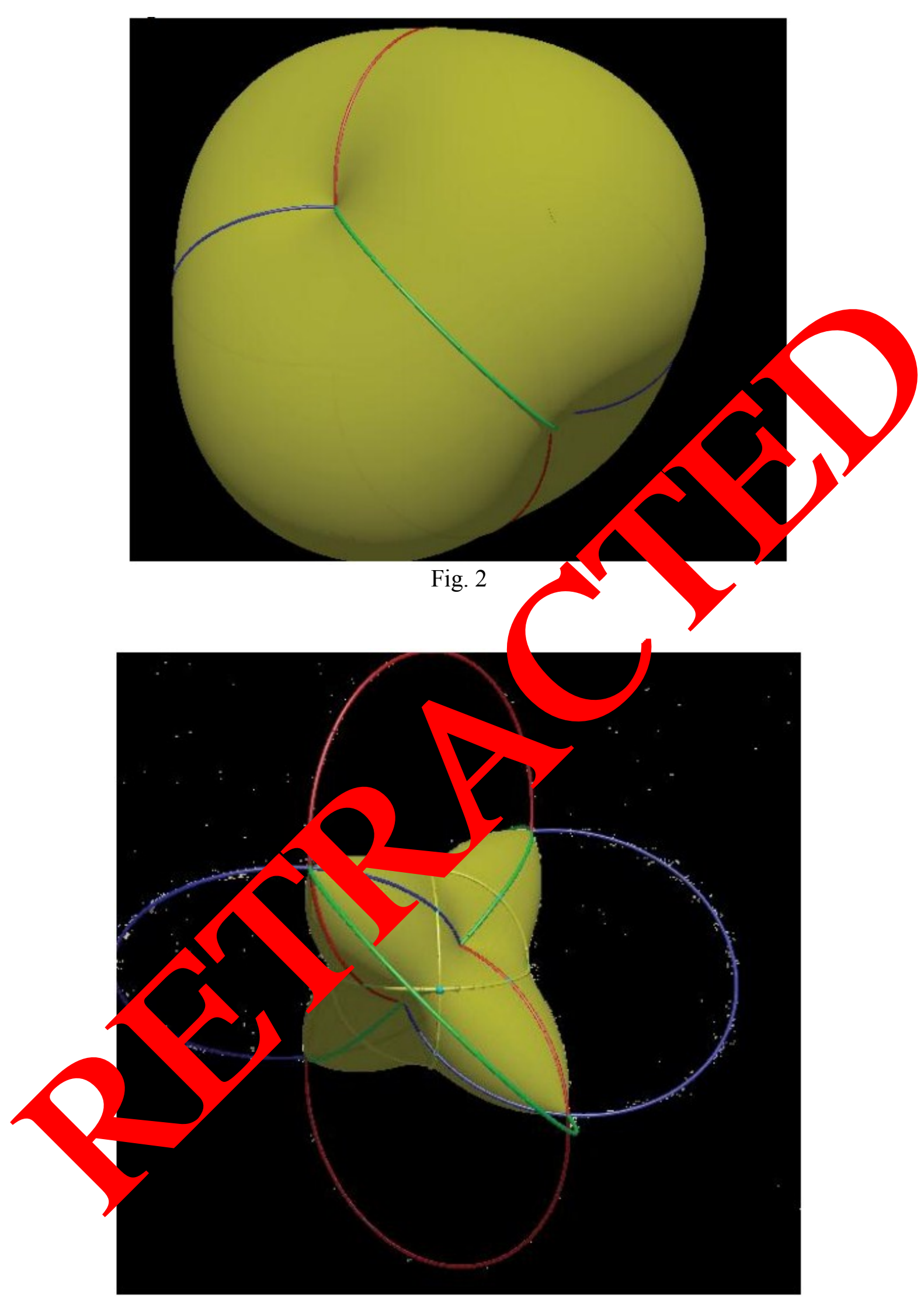

Fig. 3

The torus shown in Fig.2 is due to Marcelis [13] whose calculations in a projective space with 24 vertices appear to be unpublished, but are supported to some extent by Westy [18] (from the same school) who provides a color-coded complex map of the Riemann surface $\mathrm{z}$ that incorporates the phases of $\omega=120$ degrees. 
Murasagi [14] Ch.7 shows that Fig. 2 is a trefoil $(3,2)$ on a torus when we choose 3 points on the ends of a cylinder that can be joined to form the torus. This brings us to the goal of this contribution which is to relate the elementary particles to knots or loops in a 6-space. Here we will be guided by the work of Coxeter [5,6] who specifically labels the vertices of the torus appearing in Fig. 1 by $0, \pm 1, \omega, \omega^{2}$ where $\omega=\exp (2 \mathrm{i} \pi / 3)$ so that a knot crosses the longitude of a torus at $\omega=120$ degrees. Essentially this is a Galois Field GF(4) with permutations of $\omega$ raised to the powers $0,1,2,3$ that will be considered in more detail in the next Section where we will show how 3 quarks in 6 -space unite to become a nucleon in the projective space $\mathrm{CP}^{3}$ which collapses to $\mathrm{P}^{3}$ immediately after the Big Bang .Section 3 will employ the color-coding of Fig.1 as a model for Quantum Chromodynamics or QCD. Finally according to Rovelli [15] knots or loops in the 6-space described by $\mathrm{E}_{6}$ employed by Coxeter may also describe Loop Quantum Gravity although details are beyond the sam of this contribution. Also Arvin [2] has considered knots on a torus as a model for element ay part but excluding quarks. Again, Sundance O Billson Thompson, Smolin et.al. [17] al use knot s a model for Quantum Gravity and the Standard Model but utilize trinions instear of tr

\section{Coxeter Algebra}

Fig. 1 is a torus taken from Coxeter [5] which is an alternative to the $\operatorname{vra} 13) \mathrm{su}(3)_{\mathrm{spin}} \times$ $\mathrm{su}(3)$ isospin which is a triality sub-algebra of $\mathrm{E}_{6}$. This was publis ed later as $\mathrm{F}^{\prime}$ g.12.3B. Both graphs are orbifolds with 27 vertices that according to Slansky $\Lambda$ vay be las d by particles in the Standard Model or SM. However the actual labeling of the ritan planes on a cubic surface (discussed by Hunt [10] Ch. 4) is new, but in line with Co ceter labels. Xample the up-quark u in Fig. 1 is labeled by $(012)$ indicated by $0, \omega, \omega^{2}$. Thu $(023)$ on the sane tritangent is simply a rotation through $\omega=120$ degrees and so on. In this way $e$ find an eq ilateral triangle labeled by the 3 quarks uud comprising a proton and another ddu for t eutron b ginning with (120). There are 2 more tritangents (not labeled) for the anti-panticles whi sute the outer ring of Fig.1. But quarks also belong to a GF(4) ring and thus toil on a torus which is precisely the model adopted by Green, Schwarz and Witten [8] Secti 197.

The quarks at the vertices of Fig.1 trefoils 1lustrated by Fig.2, but the torus in Fig.1 only becomes a trefoil after the collapse of th inner ri yust after the Big Bang when quarks in the 6space $\mathrm{CP}^{3}$ unite to build nucleon the $\mathrm{p}$ biective pace $\mathrm{P}^{3}$.

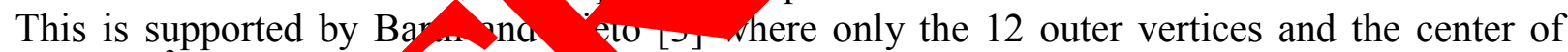
Fig. 1 are in $\mathrm{P}^{3}$.Specifically ese autho 'nd 15 synthemes, where a syntheme has 6 'fix-lines' that are the edges of an inv lan trahedron uch as u u d 0 representing a proton. However because there are only 3 ver on the of a syntheme the outer ring of Fig. 1 carries the 4 stable particles proton, eutren and the anti-particles. Also since the tritangents are invariant under rotations ther re as lly $3 \times 4=12$ possible synthemes on the outer ring. Specifically each syntheme consists 3 comn ting operators. Thus 3 synthemes can be chosen for spin rotations about the so of ace Thus introducing triality which is a characteristic of Toric-Calabi-Yau modul spac that car the Hessian Polyhedra in $\mathrm{E}_{6}$ as discussed by Lie-Yang [12] and analysed by Cox

Spec Jly each rotation in 3-space is also accompanied by a corresponding rotation in a parity 4-spa 1 en we permute 1,2,3. Charge conjugation belongs to a second set of 3 synthemes with the samerotations in a 3 -space but a parity 5-space in another charge space [7].

In this way the 12 unstable particles sss, overline (sss), $\mu^{ \pm}, v_{\mu} ; \tau^{ \pm}, v_{\mathrm{r}}$ do not appear in the compactification of $C P^{3}$ to $P^{3}$. This may be visualised as a collapse of the inner vertices to a point which carries the remaining 3 synthemes $e^{ \pm}, v_{e}$. labeled by $\{011,022,033\},\{110,220,330\}$ and $\{101,202,303\}$ for the muon. In this process the masses $m_{\square} m_{v}$ of the $\tau, \mu$ reappear as stable deuterium 3

according to the relationship

$$
m_{\tau}+m_{\mu}=m_{p}+m_{n}+m_{e}
$$


There is no heavy-ion decay and the same relation holds for the anti-particles. This equation is accurate if we assume that $\mathrm{m}_{\tau}=1777 \mathrm{MeV}$ and $\mathrm{m}_{\mu}=101.4 \mathrm{MeV}$ instead of the Fermi decomposition of muon decay in the weak interaction yielding $106 \mathrm{MeV}$. However in a recent publication Benjamin Brau et.al.[4] find a value of approximately $100 \mathrm{MeV}$ for the mass of cosmicray muons so there is as yet some experimental uncertainty.

\section{Quantum Chromodynamics,QCD}

Returning again to Fig.1, when the inner vertices are contracted to a point at the origin the red, green and blue lines could serve as gluons on a new torus where a red upper path passes through the center before emerging at the circumference and giving way to a green gluon that in turn passes under the torus and then over to connect with a down quark and so on. The 3 colom dimensions vanish when $\mathrm{CP}^{3} \rightarrow \mathrm{P}^{3}$ but a torus knot remains in 3-space.

However Marcelis [13] calculates the dual set of 3 paths for the anti-gluons verli $(r, g, b)$ y lich appear in Fig.3 ( without the quadrupole) so the gluon, antigluon linked tre oil give u. $S J(3)_{c}$ color symmetry underlining QCD as described by Griffiths [9],Sect 9.1. exan when another quark is added after a rotation $\omega$ a red gluon may unite with $n$ a tue to ild $r$ anti-b, then a following rotation would bring $r$ to anti-r and so on before low dow P3 1 this way we can find 9 gluon pairs $r$ anti-r, $r$ anti- $b$, r anti- $g$; b anti-r,b anti-,$b$ i- g;g ant, g anti-b,g anti- $g$ that are a basis for $\mathrm{SU}(3)_{\mathrm{c}}$ symmetry.

Finally Adams [1] p 273 also envisages the 3 colors r,b pas ree extra censions in a 6-space

\section{Acknowledgement}

This contribution is a revision of a paper alrea ${ }^{-}$submitto $_{\text {a }}$ SA $178 \mathrm{X}$ that was unfortunately published without the Figures

Fig.1 The Coxeter Polytope

Fig. 2 Cayley Surface in Elliptic Sp ce

Fig.3 Interior of Cayley Surface

4 Bibliography

References

[1] C.C. Adam The K Book, Freeman, 1994.

[2] J.S.Arvin, K. on a To s: A Model of Elementary Particles, symmetry,4(2013)39-115.

[3] W.B and 1. to, Abelian Surfaces of Type (1,3) and Quartic Surfaces, J. Algebraic Geome ty $3($ 94)173 - Benjamin Brau, Determining the Muon Mass in an Instructional Labora

[4] Ben, in Brau, Determining the Muon Mass in an Instructional Laboratory, Am.J.Phys. 73(2010)64- Xiv:0907.5641.

[5] H.S.M.C xeter, The Polytope 221 whose 27 Vertices Correspond to the Lines on the General Cubic Surface, Am. Mathematical Soc.62(1940)467486.

[6] H.S.M.Coxeter, Regular Complex Polytopes, Camb.Univ.Press, Second Edition(1991).

[7] J.A.de Wet, Icosahedral Symmetry in the MSSM, International Mathematical Forum 5(2010) 291-300. Available on-line under Hikari,Ltd.

[8] M.B.Green,J.H.Schwarz and E.Witten, Superstring Theory ,Camb.Univ.Press (1998).

[9] David Griffiths, Introduction to Elementary Particles, WILEY-VCH(2004).

[10] H.Kedia, I.Bialynicki, D.Peralta-Salas and William Irvine, Tying Knots in LightFields,arXiv:1302.0342. 
[11] Bruce Hunt, The Geometry of Some Arithmetic Quotients, Lecture Notes in mathematics, 1637,Springer(1996).

[12] Lei-Yang, Hessian Polyhedra Invariant Theory and Appell Hypergeometric Partial Differential Equations, arXiv:math/0412065v2.

[13] http://members.home.nl/fg.marcelis/ellip-cayley.html.

[14] Kunio Murasugi, Knot Theory and its Applications, Birkhauser, Boston(1996).

[15] Carlo Rovelli, Loop Quantum Gravity, arXiv:gr-qc/971008.

[16] R.Slansky, Group Theory for Unified Model Building, Reprinted in 'Unity of Forces in the Universe' Ed.A.Lee, World Scientific(1997).

[17] Sundance O.Billson Thompson ,Fotini Markopoulos and Lee Smolin, Quantum Gravity and the Standard Model, On-Line (2014)

[18] Westy, Platonic Tesselations of Riemann Surfaces, http:/westy31.xs4all.nl/Geometry/Geometry.html.

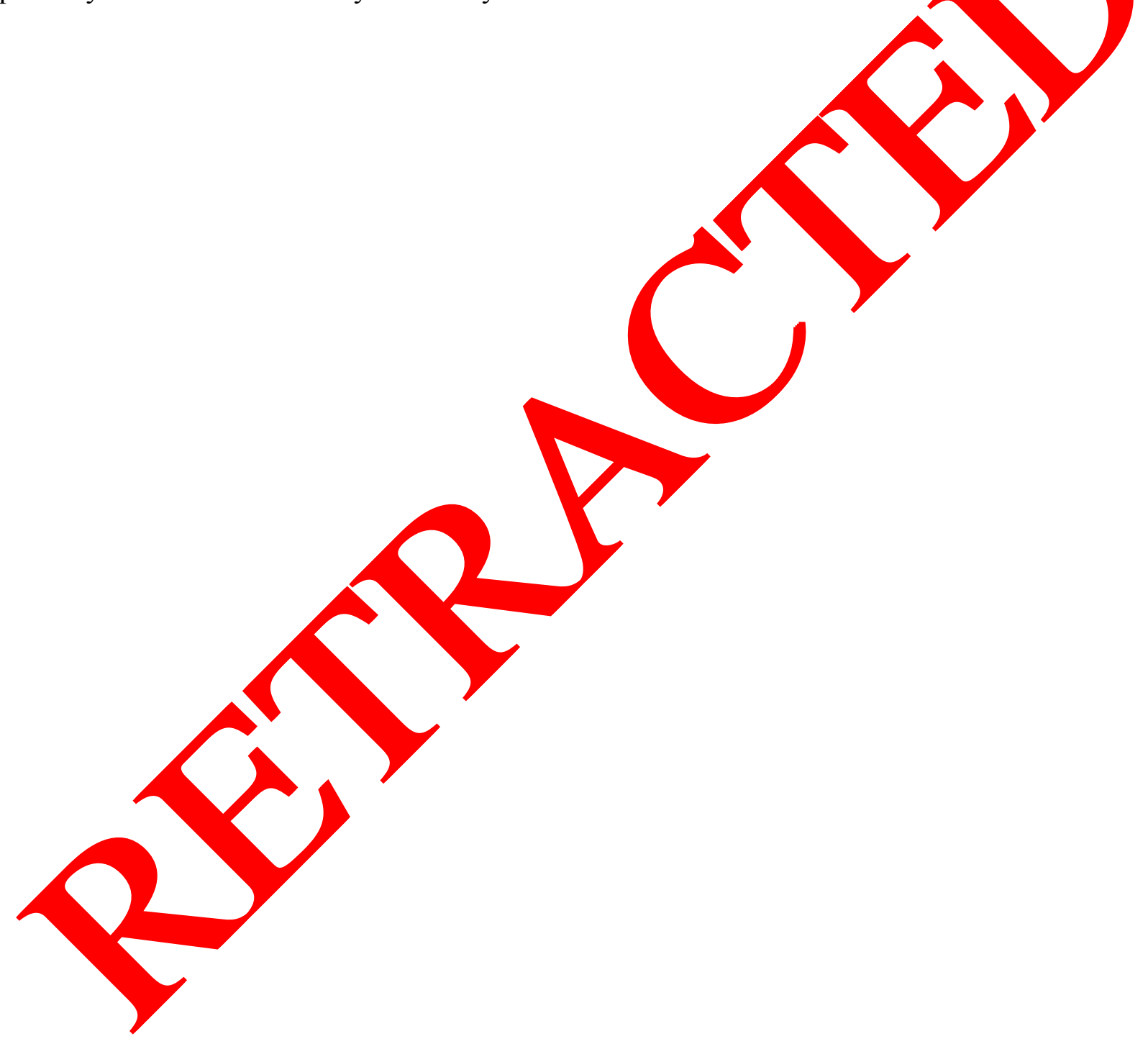

